\title{
WIRELESS TRANSMISSIONS WITH COMBINED GAIN RELAYS OVER FADING CHANNELS
}

\author{
Theodoros A. Tsiftsis \\ Dept. of Electrical \& Computer Engineering, University of Patras, \\ Rion, 26500 Patras, Greece \\ tsiftsis@ee.upatras.gr
}

George K. Karagiannidis

Dept. of Electrical \& Computer Engineering, Aristotle University of Thessaloniki, 54124 Thessaloniki, Greece

geokarag@auth.gr

Stavros A. Kotsopoulos

Dept. of Electrical \& Computer Engineering, University of Patras, Rion, 26500 Patras, Greece

kotsop@ee.upatras.gr

\begin{abstract}
We present a dual-hop relayed wireless communication system where the gain of the relay, called combined gain relay (CGR), is produced after combining the channel state information from both hops, depending on the mean hop's signal-to-noise ratio (SNR). The proposed scheme can be efficiently applied in dual-hop transmissions with unbalanced mean SNRs due to the long-term fading effects produced by the movement of the user in the area served by the wireless network. The overall system performance is studied in Rayleigh fading channels. Closed-form expressions are derived for important system performance metrics, such as average end-to-end SNR, average error probability and outage probability. Furthermore, we investigate the CGR's average power consumption which in certain cases is lower compared to existed relays. Numerical results and simulations show an improvement in the end-to-end system performance.
\end{abstract}

Keywords: Average bit error probability (ABEP), combined gain relay (CGR), dual-hop transmissions, outage probability, Rayleigh fading. 


\section{Introduction}

Multihop relaying technology is a promising solution for the throughput and high data rates coverage requirements, in future cellular and ad-hoc wireless communication systems without the need to use large power at the transmitter, and to combat fading and shadowing in wireless channel through spatial/multiuser diversity [1], [2], [3]. Nowadays, there is a great interest in the research community on the potential of multihop and especially on dual-hop transmissions [4], [5], [6], [7].

Looking through the recent up-to-date open technical literature, the performance of dual-hop wireless communication systems are studied in [1], [4], [5], [6], [7]. Hasna and Alouini have presented a useful and semi-analytical framework for the evaluation of the end-to-end outage probability of multihop wireless systems with non-regenerative channel state information (CSI)-assisted relays over Nakagami- $m$ fading channels [1]. Moreover, the same authors have studied the outage and the error performance of dual-hop systems with regenerative and non-regenerative CSI-assisted relays over Rayleigh [4] and Nakagami- $m$ [5] fading channels. The analysis in [1], [4], and [5] is based on an upper bound for the end-to-end signal-to-noise ratio (SNR) which leads to lower bounds for the system's outage and average error probability. This bound corresponds to an ideal relay capable of inverting the channel in the previous hop (regardless of the fading state of that hop) without limiting the output power. Furthermore, in [6], the end-to-end performance of dual-hop systems equipped with non-regenerative fixed gain relays is investigated and a specific relay is proposed, called semi-blind, that benefits from the knowledge of the first hop's average fading power. Recently, Tsiftsis et al. presented a new upper bound for the end-to-end SNR and efficiently evaluated the average error probability in dual-hop collaborative diversity systems, especially at low SNRs [7]. Finally, Anghel and Kaveh in [3] have studied the error performance of a cooperative network of dual-hop transmissions with parallel CSI-assisted relays in Rayleigh fading, where multi-user spatial diversity is used to combat the signal's impairments.

In this paper, we present a dual-hop transmission system, where the gain of the relay, called combined gain relay (CGR), is produced using CSI from both hops, depending on the mean hop's SNR. The proposed scheme can be efficiently used in dual-hop wireless transmissions with unbalanced mean SNRs between the hops due to the long-term fading effects produced by the movement of the user in the area served by the wireless network. The overall system performance is studied in Rayleigh fading channels as follows: Closed-form expressions for the moments, the average bit error probability (ABEP) and the outage probability of the end-to-end SNR are derived. Furthermore, we study the average power consumed by the CGR and it is shown that in certain cases it 
is less compared to existed relays. Moreover, numerical examples and Monte Carlo simulations show that CGR results in a significant improvement in the end-to-end system performance, compared to existed gain relay schemes.

\section{The Combined Gain Relay (CGR) Scheme}

\subsection{System and Channel Model}

A dual-hop wireless communication system with a non-regenerative relay operating over independent, but not necessarily identically distributed Rayleigh fading channels, is considered. The source terminal S communicates with the destination terminal $\mathrm{D}$ through the terminal $\mathrm{R}$ which acts as relay. Assuming that terminal $S$ transmits a signal with an average power normalized to unity, the received signal at terminal $R$ can be written as

$$
r_{R}=\alpha_{1} s+n_{1}
$$

where $\alpha_{1}$ is the fading amplitude of the channel between terminals $\mathrm{S}$ and $\mathrm{R}$ (first hop), modelled as a Rayleigh random variable and following the probability density function (PDF)

$$
f_{\alpha_{i}}\left(\alpha_{i}\right)=\frac{2 \alpha_{i}}{\Omega_{i}} \exp \left(-\frac{\alpha_{i}^{2}}{\Omega_{i}}\right), \quad i=1,2
$$

where $\Omega_{i}=\overline{\alpha_{i}^{2}}$ is the averaging fading power of the $i$ th hop and $n_{1}$ is the additive white Gaussian noise (AWGN) with single-sided power spectral density (PSD) $N_{0}$. The signal $r_{R}$ is then multiplied by the gain $g$ of terminal R and re-transmitted to terminal $\mathrm{D}$. The received signal at terminal $\mathrm{D}$ can be written as

$$
r_{D}=g \alpha_{2}\left(\alpha_{1} s+n_{1}\right)+n_{2}
$$

where $\alpha_{2}$ is the fading amplitude of the channel between $\mathrm{R}$ and $\mathrm{D}$ (second hop), following the PDF in (2) and $n_{2}$ is the AWGN with single-sided PSD $N_{0}$. We have omitted the time index in (1) and (3) for brevity. Using (3), the overall instantaneous SNR at the receiving end can be written as

$$
\gamma_{e n d}=\frac{\left[\alpha_{2} g a_{1}\right]^{2}}{\left[\left(\alpha_{2} g\right)^{2}+1\right] N_{0}}=\frac{\frac{\alpha_{1}^{2}}{N_{0}} \frac{\alpha_{2}^{2}}{N_{0}}}{\frac{\alpha_{2}^{2}}{N_{0}}+\frac{1}{g^{2} N_{0}}} .
$$

\subsection{Mode of Operation}

When terminal $\mathrm{R}$ has available CSI from the first hop, one kind of gain relay proposed and studied in previously published works $[8,9]$, is given by

$$
g_{1}^{2}=\frac{1}{\alpha_{1}^{2}+N_{0}} \text {. }
$$


The choice of this gain aims to limit the output power of the relay, if the fading amplitude of the first channel, $\alpha_{1}$, is low.

Next, we propose an alternative mode of operation for the relay-node $\mathrm{R}$, called combined gain relay (CGR), as follows:

Step 1: Periodically, and in synchronization with terminal D, terminal $R$ estimates the average SNR of the first hop, $\bar{\gamma}_{1}$, and terminal D estimates the average SNR of the second hop, $\bar{\gamma}_{2}$, and sends it to terminal R.

Step 2: Terminal $\mathrm{R}$ generates the new gain relay, according to the following rule:

$$
g^{2}= \begin{cases}g_{1}^{2}: 1 /\left(\alpha_{1}^{2}+N_{0}\right), & \text { if } \xi<\xi_{t h} \\ g_{2}^{2}: 1 /\left(\alpha_{2}^{2}+N_{0}\right), & \text { if } \xi>\xi_{t h}\end{cases}
$$

where $\xi=\bar{\gamma}_{1} / \bar{\gamma}_{2}$ denotes the degree of the average SNR unbalance. The parameter $\xi_{t h}$ is the threshold which signals the transition between the two available gains and depends on the performance criterion under consideration (i.e., either the average end-to-end SNR or the error probability or the outage probability). The way to choose values for $\xi_{t h}$ will be discussed in Section 5 . Note, that the choice of $g_{2}$ does not limit the instantaneous output power of the relay.

The ability of relay $\mathrm{R}$ to generate the appropriate gain, depending on the fading conditions in both hops, leads to an end-to-end SNR, which using (4) with (6) and (7) is formulated as

$$
\gamma_{e n d}= \begin{cases}\gamma_{1} \gamma_{2} /\left(\gamma_{1}+\gamma_{2}+1\right), & \text { if } \xi<\xi_{\text {th }} \\ \gamma_{1} \gamma_{2} /\left(2 \gamma_{2}+1\right), & \text { if } \xi>\xi_{\text {th }}\end{cases}
$$

where $\gamma_{i}$ is the instantaneous SNR of the $i$ th hop, following the exponential PDF defined as

$$
f_{\gamma_{i}}\left(\gamma_{i}\right)=\frac{1}{\bar{\gamma}_{i}} \exp \left(-\frac{\gamma_{i}}{\bar{\gamma}_{i}}\right), \quad i=1,2 .
$$

Note that, as mentioned in Section 1, the performance analysis of dual-hop systems with relay given by (6) has been extensively studied in the literature [1], [3], [4], [5], [7]. Thus, in the following, only the case of $g=g_{2}$ will be further studied.

\section{Performance Analysis}

\subsection{Moments of the End-to-End SNR}

The first and the second order moments of the end-to-end SNR are statistical parameters which can be efficiently used to evaluate important performance 
system measures, such as average output SNR and variance. By definition, the $n$th moment of $\gamma_{e n d}$, for $g=g_{2}$, is given by

$$
E\left\langle\gamma_{\text {end }}^{n}\right\rangle=\int_{0}^{\infty} \int_{0}^{\infty}\left(\frac{\gamma_{1} \gamma_{2}}{2 \gamma_{2}+1}\right)^{n} f_{\gamma_{1}}\left(\gamma_{1}\right) f_{\gamma_{2}}\left(\gamma_{2}\right) d \gamma_{1} d \gamma_{2}
$$

where $E\langle\cdot\rangle$ denotes expectation and substituting (10) into (11), E $\left\langle\gamma_{\text {end }}^{n}\right\rangle$ can be evaluated as (see [10])

$$
E\left\langle\gamma_{e n d}^{n}\right\rangle=\frac{(n !)^{2}}{2^{n}} \bar{\gamma}_{1}^{n} U\left(n, 0, \frac{1}{2 \bar{\gamma}_{2}}\right)
$$

where $U(\cdot, \cdot, \cdot)$ is the Kummer's function defined in [11, eq. (13.1.3)]

Average End-to-End SNR. Using (12) for $n=1$, the average end-to-end SNR can be obtained as

$$
\bar{\gamma}_{e n d}=\frac{\bar{\gamma}_{1}}{2} \exp \left(\frac{1}{2 \bar{\gamma}_{2}}\right) \mathrm{E}_{2}\left(\frac{1}{2 \bar{\gamma}_{2}}\right)
$$

where $\mathrm{E}_{k}(\cdot)$ is the exponential integral [12, eq. (5.1.4)] with $k$ being a positive integer.

\subsection{Average Symbol Error Probability (ASEP)}

The error performance, for several digital modulation schemes, can be efficiently studied using the well-known moment generating function (MGF)based approach [13]. The MGF, defined here as

$$
\mathcal{M}_{\gamma_{\text {end }}}(s) \triangleq E\left\langle\exp \left(-s \gamma_{e n d}\right)\right\rangle
$$

can be evaluated in closed-form solving the following double integral

$$
\mathcal{M}_{\gamma_{e n d}}(s)=\int_{0}^{\infty} \int_{0}^{\infty} \exp \left(-s \frac{\gamma_{1} \gamma_{2}}{2 \gamma_{2}+1}\right) f_{\gamma_{1}}\left(\gamma_{1}\right) f_{\gamma_{2}}\left(\gamma_{2}\right) d \gamma_{1} d \gamma_{2}
$$

Substituting $f_{\gamma_{i}}\left(\gamma_{i}\right)$ into (15), the first integral in (15) (i.e., the one on $\gamma_{1}$ ) is of the form

$$
\mathcal{I}_{1}=\frac{1}{\bar{\gamma}_{1} \bar{\gamma}_{2}} \int_{0}^{\infty} \exp \left(-s \frac{\gamma_{1} \gamma_{2}}{2 \gamma_{2}+1}\right) \exp \left(-\frac{\gamma_{1}}{\bar{\gamma}_{1}}\right) d \gamma_{1}
$$

which can be solved using [12, eq. (3.310)] as

$$
\mathcal{I}_{1}=\frac{1}{\bar{\gamma}_{2}} \frac{2 \gamma_{2}+1}{\left(s \gamma_{2} \bar{\gamma}_{1}+2 \gamma_{2}+1\right)}
$$


The second integral in (15) (i.e., the one on $\gamma_{2}$ ) can now be written using (17) as

$$
\mathcal{I}_{2}=\frac{1}{\bar{\gamma}_{2}} \int_{0}^{\infty} \frac{2 \gamma_{2}+1}{s \gamma_{2} \bar{\gamma}_{1}+2 \gamma_{2}+1} \exp \left(-\frac{\gamma_{2}}{\bar{\gamma}_{2}}\right) d \gamma_{2}
$$

Using [12, eq. (3.352.4)], [12, eq. (3.353.5)] and [11, eq. (5.1.45)], (18) yields to

$$
\mathcal{M}_{\gamma_{\text {end }}}(s)=\frac{2}{2+s \bar{\gamma}_{1}}+\frac{s \bar{\gamma}_{1} \exp \left(\frac{1}{2 \bar{\gamma}_{2}+s \bar{\gamma}_{1} \bar{\gamma}_{2}}\right)}{\bar{\gamma}_{2}\left(2+s \bar{\gamma}_{1}\right)^{2}} \Gamma\left(0, \frac{1}{2 \bar{\gamma}_{2}+s \bar{\gamma}_{1} \bar{\gamma}_{2}}\right)
$$

where $\Gamma(x, y)$ is the incomplete Gamma function, defined in [12, eq. (8.350.2)].

With the aid of $\mathcal{M}_{\gamma_{\text {end }}}(s)$, and using the MGF-based approach for the performance evaluation of digital modulations over fading channels, presented in [13], the error rates can be calculated directly for non-coherent binary signalling, such as BFSK and DPSK, while for other cases including $M$-QAM and $M$-PSK, single integrals with finite limits and integrands composed of elementary functions have to be readily evaluated via numerical integration.

\subsection{Outage Probability}

If $\gamma_{t h}$ is a certain specified threshold ratio, then for non-regenerative multihop transmissions the outage probability is defined as the probability that the instantaneous SNR at the final destination falls below $\gamma_{t h}$ and is expressed as

$$
P_{\text {out }}=\operatorname{Pr}\left[\gamma_{\text {end }} \leq \gamma_{t h}\right]=\int_{0}^{\infty} \operatorname{Pr}\left[\frac{\gamma_{1} \gamma_{2}}{2 \gamma_{2}+1} \leq \gamma_{t h} \mid \gamma_{2}\right] f_{\gamma_{2}}\left(\gamma_{2}\right) d \gamma_{2} \text {. }
$$

Following the same method as in [9], the above integral yields to

$$
P_{\text {out }}=1-2 \sqrt{\frac{\gamma_{t h}}{\bar{\gamma}_{1} \bar{\gamma}_{2}}} \exp \left(-\frac{2 \gamma_{t h}}{\bar{\gamma}_{1}}\right) K_{1}\left(2 \sqrt{\frac{\gamma_{t h}}{\bar{\gamma}_{1} \bar{\gamma}_{2}}}\right)
$$

where $K_{1}(\cdot)$ is the first order modified Bessel function of the second kind defined in [11, eq. $(9.6 .22)]$.

\section{Average Power Consumption}

In this section, we quantify the average gain of the relay (i.e., average power consumption) when CGR is considered. If $\tau$ is the percentage of the time when $g_{2}$ is used $\left(1-\tau\right.$ corresponds to $\left.g_{1}\right)$ then the average power consumed by the relay, $\bar{P}_{C G R}$, can be expressed by

$$
\bar{P}_{C G R}=\tau \bar{P}_{2}+(1-\tau) \bar{P}_{1}
$$

where $\bar{P}_{1}$ and $\bar{P}_{2}$ are the average power consumed by the relay, when $g_{1}$ or $g_{2}$ are used, respectively. For Rayleigh fading channels, $\bar{P}_{1}$ and $\bar{P}_{2}$ can be written 
Table 1. Evaluation of $\xi_{t h}$ for several values of $\bar{\gamma}_{1}$

\begin{tabular}{c||c}
\hline $\bar{\gamma}_{1}(\mathrm{~dB})$ & $\xi_{t h}$ \\
\hline \hline-5 & 0.6 \\
0 & 0.54 \\
5 & 0.58 \\
10 & 0.68 \\
15 & 0.84
\end{tabular}

as $[6$, eq. (15)]

$$
\bar{P}_{i}=E\left\langle g_{i}^{2}\right\rangle=\frac{e^{1 / \bar{\gamma}_{i}} \Gamma\left(0,1 / \bar{\gamma}_{i}\right)}{\bar{\gamma}_{i} N_{0}}
$$

\section{Numerical Results}

In this section, we provide several representative numerical and simulation examples illustrating the performance of the dual-hop system with CGR over Rayleigh fading channels. These results are compared with a system which uses only the gain relay given by (6). The threshold, $\xi_{t h}$, for the transition

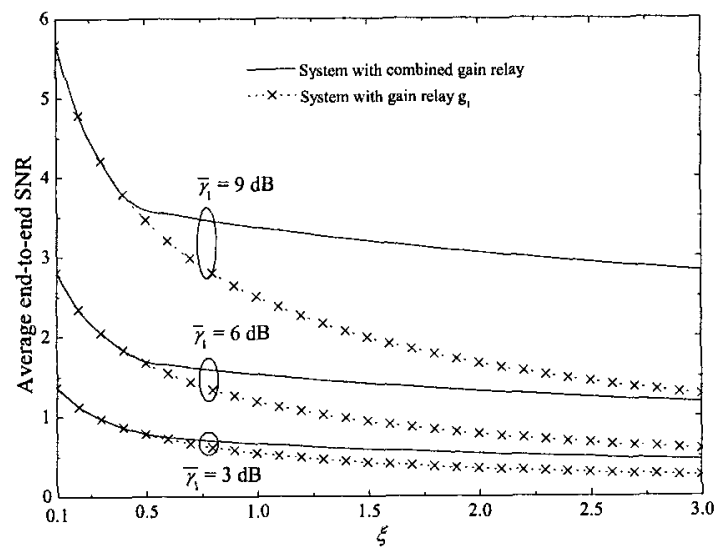

Figure 1. Comparison of the average end-to-end SNR of a dual-hop system with the relay gain $g_{1}$ and CGR versus $\xi$ for several values of $\bar{\gamma}_{1}$.

from $g_{1}$ to $g_{2}$, depends on the selected performance criterion and it can be determined after equating the formulae related to this criterion of the gains $g_{1}$ and $g_{2}$ and solving numerically with respect to $\xi$. When the criterion considered is the outage probability, then $\xi_{t h}$ can be determined by equating (21) to [9, eq. 


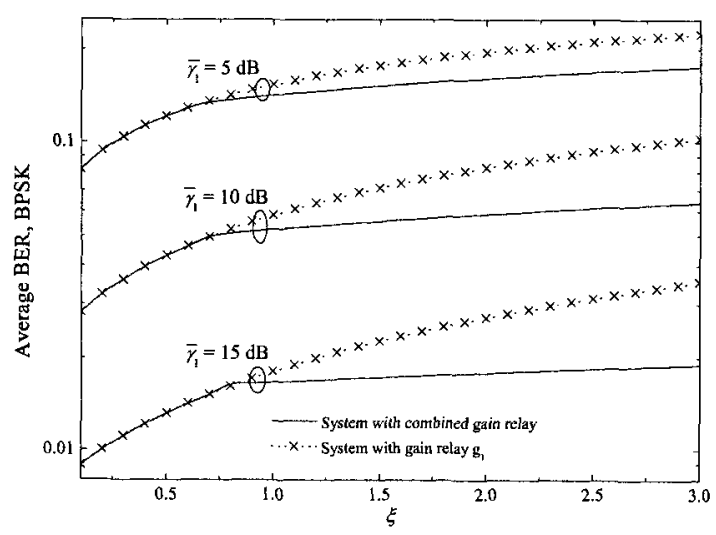

Figure 2. Comparison of the BPSK average error performance of a dual-hop system with the relay gain $g_{1}$ and CGR versus $\xi$ for several values of $\bar{\gamma}_{1}$.

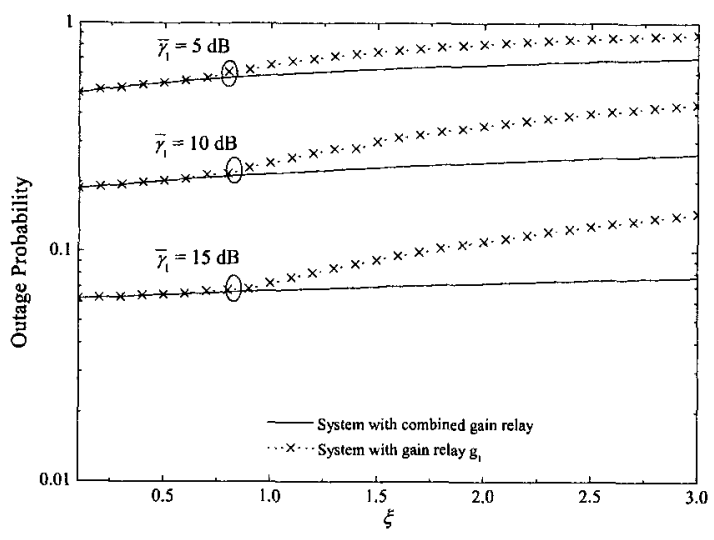

Figure 3. Comparison of the end-to-end outage probability of a dual-hop system with the relay gain $g_{1}$ and CGR versus $\xi$ for several values of $\bar{\gamma}_{1}$.

(14)] and solving numerically for $\xi_{t h}$. In Table $1, \xi_{t h}$ is evaluated for several values of $\bar{\gamma}_{1}$. It can be easily verified that almost the same values for $\xi_{t h}$ are observed when other performance criteria as the average end-to-end SNR or the ABEP are used.

In Fig. 1 the average end-to-end SNR for a dual-hop system with CGR is plotted versus $\xi$, for several values of mean SNR $\bar{\gamma}_{1}$. Monte Carlo simulations are also performed for a system using a relay with the gain $g_{1}$ and their results are depicted in the same figure. We observe that depending on the value of $\xi$, the CGR improves the overall average end-to-end SNR performance. Similar conclusions are also extracted from Figs. 2 and 3, where the BPSK average er- 


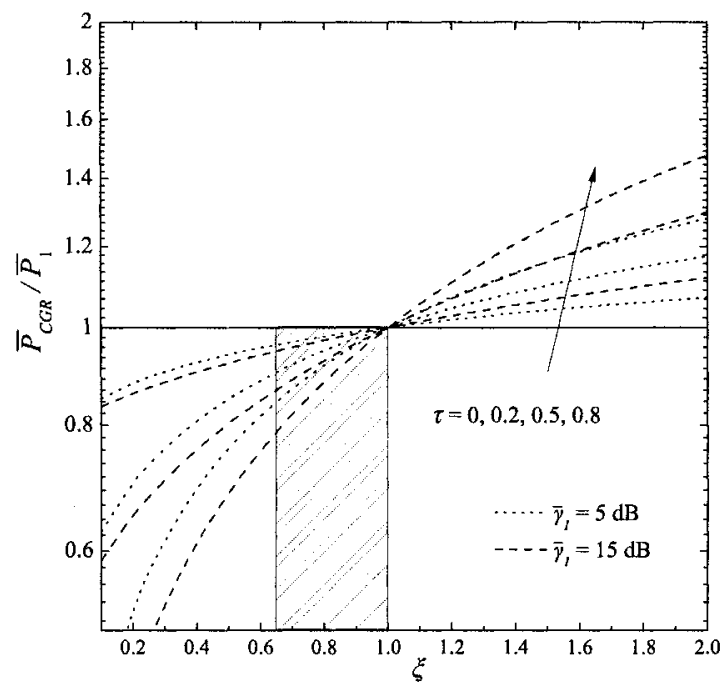

Figure 4. Ratio of the average power consumption versus $\xi$ for several values of $\tau$.

ror rate performance and the outage probability are plotted versus $\xi$ for several values of $\bar{\gamma}_{1}$, respectively.

Finally, in Fig. 4, the excess average power consumption, expressed by the ratio, $\bar{P}_{C G R} / \bar{P}_{1}$, is evaluated for several values of $\xi$. It is very interesting to observe that when $0.65 \leq \xi \leq 1$ (grey region), although the average power consumption of CGR is less than $\bar{P}_{1}$ (i.e., $\bar{P}_{C G R} / \bar{P}_{1}<1$ ), the system's performance is improved. Moreover, as expected, the average power consumption of the CGR is increased when $\xi>1$. However, by substituting (23) in (22) and using [11, eq. (6.5.31)], it can be easily verified that $\lim _{\xi \rightarrow \infty} \bar{P}_{C G R}=1 / N_{0}$.

\section{Conclusion}

A dual-hop wireless communication system with a novel combined gain relay, was presented. The new type of relay produced a gain using CSI from both hops, depending on the mean hop's SNR. This approach results to an improved end-to-end system performance compared to existed gain relays, but with an increase in average power consumption. However, it was shown that in certain cases, the average power consumed by the CGR was less compared to existed relays. Furthermore, closed-form expressions for the moments, the average error probability and the outage probability of the end-to-end SNR were derived. 


\section{References}

[1] Hasna, M.O., and Alouini, M.S. (2003). "Outage probability of multihop transmission over Nakagami fading channels," IEEE Communications Letters, vol. 7, pp. 216-218.

[2] Boyer, John., Falconer, David D., and Yanikomeroglu, Halim. (2004). "Multihop diversity in wireless relaying channels," IEEE Transactions on Communications, vol. 52, pp. 18201830.

[3] Anghel, Paul A., and Kaveh, M. (2004). "Exact symbol error probability of a cooperative network in a Rayleigh-Fading Environment," IEEE Transactions on Wireless Communications, vol. 3, pp. 1416-1421.

[4] Hasna, M.O., and Alouini, M.-S. (2003). "End-to-end performance of transmission systems with relays over Rayleigh fading channels," IEEE Transactions on Wireless Communications, vol. 2, pp. 1126-1131.

[5] Hasna, M.O., and Alouini, M.-S. (2004). "Harmonic mean and end-to-end performance of transmission systems with relays," IEEE Transactions on Communications, vol. 52, pp. 130-135.

[6] Hasna, M.O., and Alouini, M.-S. (2004). "A performance study of dual-hop transmissions with fixed gain relays," IEEE Transactions on Wireless Communications, vol. 3, pp. 19631968.

[7] Tsiftsis, T.A., Karagiannidis, G.K., Kotsopoulos S.A., and Pavlidou, F.-N. (2004). "BER analysis of collaborative dual-hop wireless transmissions," IEE Electronics Letters, vol. 11, pp. 1732-1745.

[8] Laneman, J.N., and Wornell, G.W. (2000). "Energy-efficient antenna sharing and relaying for wireless networks," in Proc. IEEE Wireless Communications and Networking Conf. (WCNC'00), Chicago, IL, pp. 7-12.

[9] Emamian, V., Anghel, P., and Kaveh, M. (2000). "Multi-user spatial diversity system in a shadow-fading environment," in Proc. IEEE Vehicular Technology Conference (Fall VTC'02), Vancouver, BC, Canada, pp. 573-576.

[10] Tsiftsis, T.A., Karagiannidis G.K., and Kotsopoulos, S.A. (2005). "Dual-hop wireless communications with combined gain relays (CGR)," accepted for publication in IEE Proc. Communications.

[11] Abramovitz M.A., and Stegun, I.A. (1972). Handbook of Mathematical Functions with Formulas, Graphs, and Mathematical Tables, 9th ed. New York: Dover.

[12] Gradshteyn, I.S., and Ryzhik, I.M. (2000). Table of Integrals, Series, and Products, 6th ed. New York: Academic.

[13] Simon, M.K., and Alouini, M.-S. (2005). Digital Communication over Fading Channels, 2th ed. New York: Willey. 